\title{
Atomic Level Characterization of Novel Hardening Mechanisms in High-Mn-Steels
}

\author{
Joachim Mayer $^{1,2}$, Maryam Beigmohamadi ${ }^{2}$, Marta Lipinska-Chwalek ${ }^{1,2}$ and James E.Wittig ${ }^{3}$ \\ ${ }^{1 .}$ Central Facility for Electron Microscopy, RWTH Aachen University, Aachen, Germany \\ 2. Ernst Ruska Centre, Forschungszentrum Jülich, Germany \\ ${ }^{3 .}$ Interdisciplinary Materials Science, Vanderbilt University, Nashville TN, 37235 USA
}

Recently developed high-manganese steels exhibit an exceptional combination of strength and ductility and show great promise for structural applications. Understanding the relationships between manganese and carbon content, microstructure, temperature, defect formation and strain-hardening behavior is critical for alloying, design, and further optimization of these steels. The present study investigates the influence of alloy content, temperature and deformation behavior on the microstructural evolution of an austenitic Fe-14Cr-16Mn-0.3C-0.3N alloy showing twinning induced plasticity (TWIP) and of a twophase nanostructured Fe-30.5Mn-8Al-1.2C alloy exhibiting microband induced plasticity (MBIP).

The twinning induced plasticity (TWIP) effect enables designing austenitic Fe-Mn-C based steels with $>70 \%$ elongation at an ultimate tensile strength $>1 \mathrm{GPa}$. These alloys are characterized by high strain hardening coefficients owing to the formation of twins and complex dislocation substructures which dynamically reduce the dislocation mean free path. Further insight in the strain hardening mechanisms can be gained by a conventional TEM analysis of highly strained samples of a stainless Fe-Cr-Mn-C-N steel [1]. The primary mechanical twins contribute to the strain-hardening by acting as obstacles to dislocations gliding on different slip systems, as illustrated in Fig. 1a, which shows mechanical twins and intersecting stacking faults after 0.21 logarithmic strain. Dislocation accumulation at the twin boundaries is evident in Fig. 1b, which is a higher magnification image of the twinned area in Fig. 1a under diffraction conditions which make the dislocation arrangement visible. High resolution TEM and STEM images of the planar defects in the same alloy are presented in Figure 2. Detailed investigations revealed insight in the atomistic structure of the defects and the defect/dislocation interaction. The presented examples reveal that TEM investigations can give important insight in the deformation mechanisms and mechanical properties of the high-Mn alloys.

In further HRTEM investigations, microband induced plasticity (MBIP) alloys with a composition of Fe-30.5Mn-8Al-1.2C were investigated after various heat treatments at $600^{\circ} \mathrm{C}$. In the alloys, regularly spaced coherent precipitates of the $\kappa$-Phase were found, which cause the unique properties of these materials. Fig. 3a shows a low magnification image of the regular arrangement of the cube- or brickshaped nanoscale $\kappa$-carbide precipitates. At higher magnifications, a coherent embedding of the precipitates in the austenitic matrix can be discerned (Fig. 3b). The specific thermal and mechanical properties of the alloys can be attributed to the mechanical strain introduced by the coherency at the matrix/precipitate interfaces (Fig. 3c). In further investigations, the microstructural evolution and the strain relaxation upon further annealing were investigated.

\section{References:}

[1] L. Mosecker, D.T. Pierce, A. Schwedt, M. Beighmohamadi, J. Mayer, W. Bleck, J.E. Wittig, Materials Science \& Engineering A 642 (2015) 71-83.

[2] Finanial support from the German Research Foundation (DFG, SFB761) is gratefully acknowledged. 


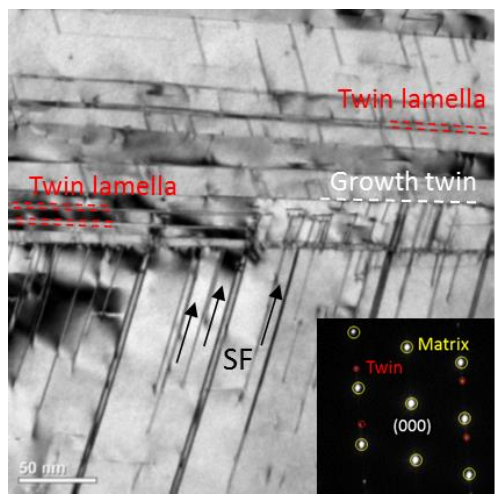

a

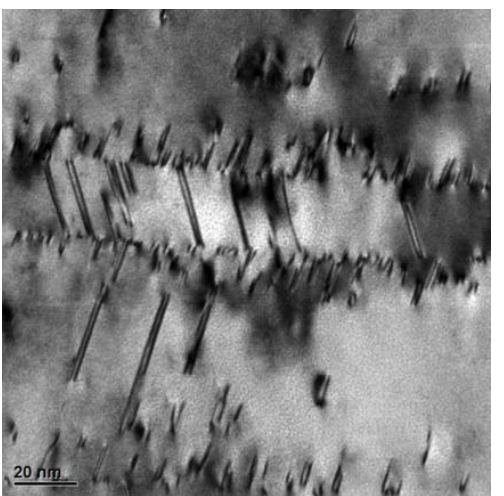

b

Figure 1. TEM investigations of an austenitic Fe-14Cr-16Mn-0.3C-0.3N alloy. (a) Bright-field image of extended stacking faults blocked at twin lamella and (b) $\langle 111\rangle$ twin plane reflection showing dislocations pile-ups at planar defects at $\varepsilon_{3}=0.21$.

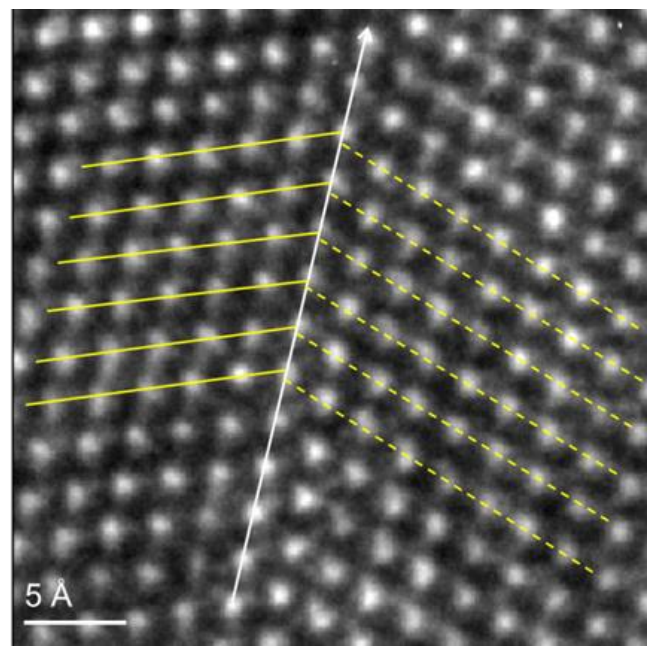

a

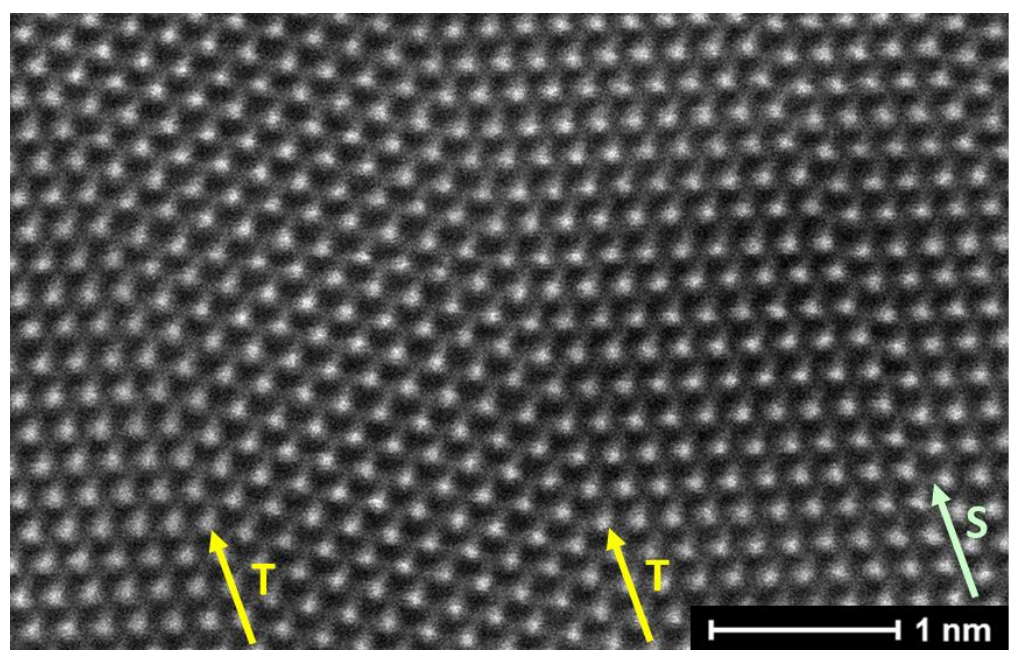

b

Figure 2. (a) HRTEM image of a single twin boundary in the austenitic Fe-14Cr-16Mn-0.3C-0.3N alloy. (b) HRSTEM image of a narrow twin (boundaries denoted by $\mathrm{T}$ ) and a stacking fault (denoted by S) in the same alloy.

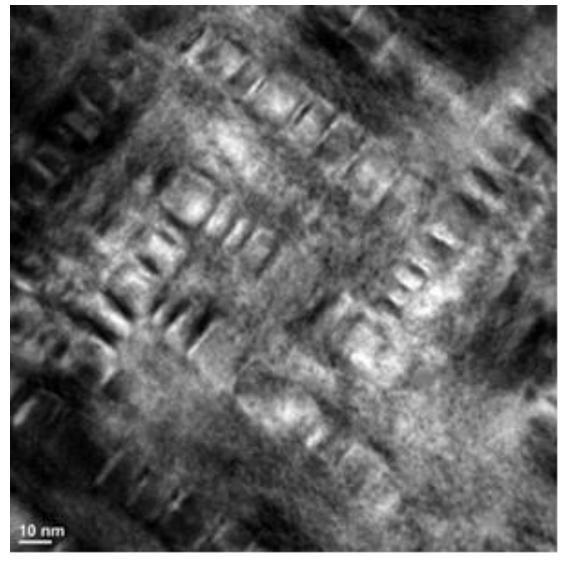

a

\section{a 3 . Conventional TEM (a)}

Figure 3. Conventional TEM (a) and HRTEM investigations (b,c) of an MBIP-alloy with a composition of $\mathrm{Fe}-30.5 \mathrm{Mn}-8 \mathrm{Al}-1.2 \mathrm{C}$ after heat treatment at $600^{\circ} \mathrm{C}$ for 1 hour. 